\title{
Chiral Phase Transitions in QCD at Finite Temperature: Hard-Thermal-Loop Resummed Dyson-Schwinger Equation in the Real Time Formalism
}

\author{
Hisao NAKKAGAWA, Hiroshi YOKOTA, Koji YOSHIDA \\ Institute for Natural Sciences, Nara University, Nara 631-8502, Japan \\ and Yuko FUEKI \\ Department of Physics, Nara Women's University, Nara 630-8506, Japan
}

\begin{abstract}
Chiral phase transition in thermal QCD is studied by using the DysonSchwinger (DS) equation in the real time hard thermal loop approximation. Our results on the critical temperature and the critical coupling are significantly different from those in the preceding analyses in the ladder DS equation, showing the importance of properly taking into account the essential thermal effects, namely the Landau damping and the unstable nature of thermal quasiparticles.
\end{abstract}

Although lots of efforts have been made to undestand the temperature/density-dependent phase transition in thermal QCD, we still have not really understood yet even the relation between the chiral and the deconfinement phase transitions. The analysis with the use of DysonSchwinger (DS) equation is a powerful tool to investigate with the analytic procedure the phase structure of gauge theories. At finite temperature/density, however, we should seriously ask whether the preceding results [1] of the DS equation analysis on the chiral phase transition could be relied on being the real consequences of thermal gauge theories. This is because in previous analyses the lessons from vacuum theories have been applied without close examination, having missed the essential thermal effects, thus causing the neglection of large contributions to the DS equation unless otherwise existed.

We here make a re-analysis by studying the hard-thermal-loop (HTL) resummed DS equation in the real time formalism, giving a new understanding on the phase structure and the mechanism of phase transition in thermal gauge theories. Main interest of the present investigation lies in clarifying what are the essential temperature effects that govern the phase transition and also in finding how we can closely take these effects into the "kernel" of the DS equation. Essential procedures of our analysis can be summerised as follows;

i) We use the real time closed-time-path formalism and study the physical mass function $\Sigma_{R}$ itself, not the $\Sigma_{11}$, of the retarded fermion propagator. $\Sigma_{R}$ is the mass function of "unstable" quasi-particle in thermal field theories, thus having non-trivial imaginary parts as well as nontrivial wave-function renormalization constants.

ii) To closely estimating the dominant temperature effects we focus on studying the DS equation being exact up to HTL approximation: both the gauge boson propagator and the vertex functions are determined within the HTL approximation, guaranteeing the gauge invariance of the result at least in the perturbative analysis. With the HTL vertex functions [2] we can derive the desired HTL resummed DS equation.

The second point listed above is better to be taken step by step into the actual analysis of the DS equation. In the present paper we present the result of our first step investigation; focussing on what happens when taking into account exactly the HTL resummed gauge 
boson propagators. We show explicitly the results of preceding analyses can not be the real consequences of thermal gauge field theories.

Decomposing the fermion mass function $\Sigma_{R}$ as

$$
\Sigma_{R}(P)=(1-A(P)) p_{i} \gamma^{i}-B(P) \gamma^{0}+C(P),
$$

with $A(P), B(P)$ and $C(P)$ being the three independent scalar invariants to be determined, the DS equation we here investigate in the point vertex approximation with the exact HTL resummed gauge boson propagator becomes as $\left(P^{\mu}=\left(p_{0}, \mathbf{p}\right)\right)$,

$$
\begin{aligned}
& -p^{2}[1-A(P)]=-g^{2} C_{F} \int \frac{d^{4} K}{(2 \pi)^{4}}\left[\left\{1+2 n_{B}\left(p_{0}-k_{0}\right)\right\} \operatorname{Im}\left[{ }^{*} G_{R}^{\rho \sigma}(P-K)\right] \times\right. \\
& {\left[\left\{K_{\sigma} P_{\rho}+K_{\rho} P_{\sigma}-p_{0}\left(K_{\sigma} g_{\rho 0}+K_{\rho} g_{\sigma 0}\right)-k_{0}\left(P_{\sigma} g_{\rho 0}+P_{\rho} g_{\sigma 0}\right)+p k z g_{\sigma \rho}\right.\right.} \\
& \left.+2 p_{0} k_{0} g_{\sigma 0} g_{\rho 0}\right\} \frac{A(K)}{\left[k_{0}+B(K)+i \epsilon\right]^{2}-A(K)^{2} k^{2}-C(K)^{2}}+\left\{P_{\sigma} g_{\rho 0}+P_{\rho} g_{\sigma 0}\right. \\
& \left.\left.-2 p_{0} g_{\sigma 0} g_{\rho 0}\right\} \frac{k_{0}+B(K)}{\left[k_{0}+B(K)+i \epsilon\right]^{2}-A(K)^{2} k^{2}-C(K)^{2}}\right]+\left\{1-2 n_{F}\left(k_{0}\right)\right\} \times \\
& { }^{*} G_{R}^{\rho \sigma}(P-K) \operatorname{Im}\left[\left\{K_{\sigma} P_{\rho}+K_{\rho} P_{\sigma}-p_{0}\left(K_{\sigma} g_{\rho 0}+K_{\rho} g_{\sigma 0}\right)-k_{0}\left(P_{\sigma} g_{\rho 0}+P_{\rho} g_{\sigma 0}\right)\right.\right. \\
& \left.+p k z g_{\sigma \rho}+2 p_{0} k_{0} g_{\sigma 0} g_{\rho 0}\right\} \frac{A(K)}{\left[k_{0}+B(K)+i \epsilon\right]^{2}-A(K)^{2} k^{2}-C(K)^{2}} \\
& \left.\left.+\left\{P_{\sigma} g_{\rho 0}+P_{\rho} g_{\sigma 0}-2 p_{0} g_{\sigma 0} g_{\rho 0}\right\} \frac{k_{0}+B(K)}{\left[k_{0}+B(K)+i \epsilon\right]^{2}-A(K)^{2} k^{2}-C(K)^{2}}\right]\right] \text {, } \\
& -B(P)=-g^{2} C_{F} \int \frac{d^{4} K}{(2 \pi)^{4}}\left[\left\{1+2_{B}\left(p_{0}-k_{0}\right)\right\} \operatorname{Im}\left[{ }^{*} G_{R}^{\rho \sigma}(P-K)\right] \times\right. \\
& {\left[\left\{K_{\sigma} g_{\rho 0}+K_{\rho} g_{\sigma 0}-2 k_{0} g_{\sigma 0} g_{\rho 0}\right\} \frac{A(K)}{\left[k_{0}+B(K)+i \epsilon\right]^{2}-A(K)^{2} k^{2}-C(K)^{2}}\right.} \\
& \left.+\left\{2 g_{\rho 0} 2 g_{\sigma 0}-g_{\sigma \rho}\right\} \frac{k_{0}+B(K)}{\left[k_{0}+B(K)+i \epsilon\right]^{2}-A(K)^{2} k^{2}-C(K)^{2}}\right]+\left\{1-2 n_{F}\left(k_{0}\right)\right\} \times \\
& { }^{*} G_{R}^{\rho \sigma}(P-K) \operatorname{Im}\left[\frac { A ( K ) } { [ k _ { 0 } + B ( K ) + i \epsilon ] ^ { 2 } - A ( K ) ^ { 2 } k ^ { 2 } - C ( K ) ^ { 2 } } \left\{K_{\sigma} g_{\rho 0}+K_{\rho} g_{\sigma 0}\right.\right. \\
& \left.\left.\left.-2 k_{0} g_{\sigma 0} g_{\rho 0}\right\}+\frac{k_{0}+B(K)}{\left[k_{0}+B(K)+i \epsilon\right]^{2}-A(K)^{2} k^{2}-C(K)^{2}}\left\{2 g_{\rho 0} 2 g_{\sigma 0}-g_{\sigma \rho}\right\}\right]\right] \text {, } \\
& C(P)=-g^{2} C_{F} \int \frac{d^{4} K}{(2 \pi)^{4}} g_{\sigma \rho}\left\{1+2_{B}\left(p_{0}-k_{0}\right)\right\} \operatorname{Im}\left[{ }^{*} G_{R}^{\rho \sigma}(P-K)\right] \times \\
& {\left[\frac{C(K)}{\left[k_{0}+B(K)+i \epsilon\right]^{2}-A(K)^{2} k^{2}-C(K)^{2}}+\left\{1-2 n_{F}\left(k_{0}\right)\right\} \times\right.} \\
& \left.{ }^{*} G_{R}^{\rho \sigma}(P-K) \operatorname{Im}\left[\frac{C(K)}{\left[k_{0}+B(K)+i \epsilon\right]^{2}-A(K)^{2} k^{2}-C(K)^{2}}\right]\right],
\end{aligned}
$$

where the cutoff scale $\Lambda$ is introduced, regularizing the integral. ${ }^{*} G_{R}^{\rho \sigma}(K)$ in Eqs.(2-4) is the HTL resummed retarded gauge boson propagator [3],

$$
{ }^{*} G_{R}^{\rho \sigma}(K)=\frac{1}{* \Pi_{T}^{R}-K^{2}-i \epsilon k_{0}} A^{\rho \sigma}+\frac{1}{* \Pi_{L}^{R}-K^{2}-i \epsilon k_{0}} B^{\rho \sigma}-\frac{\xi}{K^{2}+i \epsilon k_{0}} D^{\rho \sigma},
$$

where ${ }^{*} \Pi_{T / L}^{R}(K)$ being the HTL resummed transverse/longitudinal mass function [4], and $A^{\rho \sigma}=g^{\rho \sigma}-B^{\rho \sigma}-D^{\rho \sigma}, B^{\rho \sigma}=-\tilde{K}^{\rho} \tilde{K}^{\sigma} / K^{2}, D^{\rho \sigma}=K^{\rho} K^{\sigma} / K^{2}$, being the projection tensors. In the above, $\tilde{K}=\left(k, k_{0} \hat{\mathbf{k}}\right), k=\sqrt{\mathbf{k}^{2}}$ and $\hat{\mathbf{k}}=\mathbf{k} / k$. 
The DS equation, Eqs.(2-4), is still quite tough to be attacked, forcing us further approximations for the analysis to be effectively carried out. However, the approximation made use of must be consistent with the HTL approximation, without missing the important thermal effects out of the kernel of the DS equation.

Here it is worth noticing that the instantaneous exchange (IE) approximation frequently used in the preceding analyses [1] is not compatible with the HTL approximation in the strict sense. In the exact IE-limit the HTL resummed transverse mass function, ${ }^{*} \Pi_{T}^{R}(K)$, vanishes and the transverse (magnetic) mode becomes totally massless. Namely the IE approximation discards the important thermal effect coming from the Landau damping, thus dismissing the dynamical screening of the magnetic mode, causing the famous quadratic divergence of the Rutherford scattering cross section. This can be clearly seen by taking the IE-limit of the DS equation, Eqs.(2-4), and neglecting $\operatorname{Im}[A(P)]$ and $\operatorname{Im}[C(P)]$, then obtaining the following equation for $\operatorname{Im}[B(P)]\left(E \equiv \sqrt{(\mathbf{p}-\mathbf{k})^{2}}\right)$,

$$
\operatorname{Im}[B(P)]=\frac{g^{2} C_{F}}{4 \pi} m_{g}^{2} T \int_{0}^{\infty} k^{2} d k \int_{-1}^{1} d z\left(\frac{1}{E\left[E^{2}+m_{g}^{2}\right]^{2}}+\frac{1}{E^{5}}\right),
$$

showing $\operatorname{Im}[B(P)]$ to be quadratically divergent. The reason why in the previous analyses this divergence problem did not appear is that the imaginary part of $\Sigma_{R}$ is completely neglected there from the beginning, namely the equation for $\operatorname{Im}\left[\Sigma_{R}\right]$ is totally discarded.

Taking the above into account the approximation we further make use of is the IE approximation to the longitudinal gauge boson propagator, by keeping the exact HTL resummed transverse propagator. In the IE-limit the HTL resummed longitudinal mass function, ${ }^{*} \Pi_{L}^{R}(K)$, has a nonvanishing thermal Debye screening mass $m_{g}^{2} \sim(g T)^{2}$, thus even in the IE limit the longitudinal mode can take into account the essential thermal effect. In the present analysis the gauge is fixed to the Landau gauge $(\xi=0)$.

Now we solve the DS equations Eqs.(2-4) with the IE approximation to the longitudinal mode, using the iterating procedure with suitable trial functions for the solution. This method is useful so long as the convergence of the iteration is guaranteed.

At each iteration, the three-fold integration is performed over $k, k_{0}$ and $z=\cos \theta, \theta=\hat{\mathbf{p}} \cdot \hat{\mathbf{k}}$. The integration kernel of the present DS equation shows a little bit singular behavior, and the numerical integration of such a singular integrand needs careful integration prescription, which is properly managed. As a result we performed the 1000 times iterations, obtaining fairly stable solutions.

Result of the present analysis shows that the wave function renormalization constants receive 10-20 percent corrections, indicating the necessity of gauge-parameter dependent analysis. The generated size of the imaginary part is nearly the same as that of the real part, indicating the existence of the non-trivial imaginary parts, which is as expected though completely neglected in the previous analyses.

Let us now show the behavior of the mass function at some fixed value of $p$ as a function of the parameters $\alpha=g^{2} C_{F} / 4 \pi$ and $T$. The $T$ dependence of the mass function $\operatorname{Re}[C(P)]$ for various fixed values of $\alpha$, and the $\alpha$ dependence of $\operatorname{Re}[C(P)]$ for various fixed values of $T$ are shown in Figs.1a and $1 \mathrm{~b}$, both with $p_{0}=0$ and $p=0.1 \Lambda$. The errors resulting from the fluctuations are smaller than the size of the symbol used for each sample point in Figs.1a and 1b. From these figures we can see the two facts; i) The chiral phase transition is of second order, since a fermion mass is generated at a critical value of the temperature $T$ or at the critical coupling constant $\alpha$ without any discontinuity, and ii) the critical temperature $T_{c}$ at fixed value of $\alpha$ is significantly higher than the previous results [1], namely the restoration of chiral symmetry occurs at higher temperature than previously expected. Our result shows that 
as $T$ becomes smaller the critical coupling constant $\alpha_{c}$ also becomes smaller, being consistent with the zero temperature result, where the critical coupling constant $\alpha_{c}^{0}=\pi / 3$. The second fact clearly shows that in the previous analyses the important temperature effects are neglected due to the inappropriate approximations.

Present result shows the correctness of our research-strategy, thus the importance of the full HTL resummed DS equation analysis of the chiral phase transition at finite temperature/density, which is now under investigation.
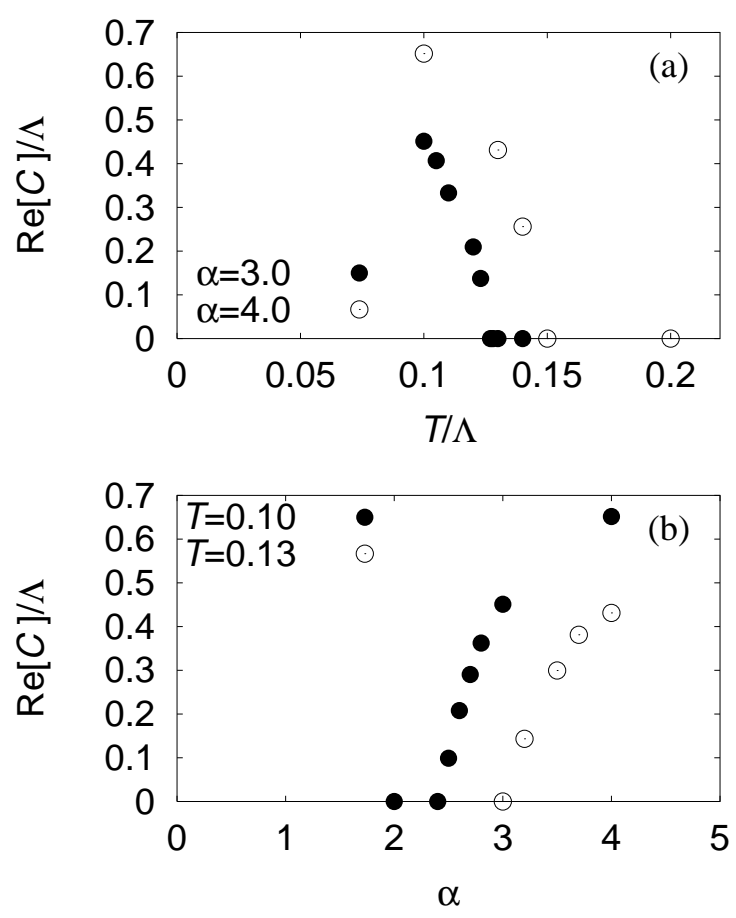

Figure 1:

\section{References}

[1] K. Fukazawa, T. Inagaki, S. Mukaigawa and T. Muta, Prog. Theor. Phys. 105, 979 (2001), and references therein.

[2] As for the real time expression, see, Y. Fueki, H. Nakkagawa, H. Yokota and K. Yoshida, hep-ph/0111275, submitted for publication.

[3] H. A. Weldon, Ann. Phys. (N.Y.) 271, 141 (1999).

[4] V. V. Klimov, Sov. J. Nucl. Phys. 33,934 (1981); Sov. Phys. JETP 55, 199 (1982); H. A. Weldon, Phys. Rev. D26, 1394 (1982); Phys. Rev. D26, 2789 (1982). 